\title{
CORRESPONDENCE
}

\section{Interhospital critical care transports: a safe trip indeed!}

\author{
E. J. van Lieshout, N. P. Juffermans ${ }^{*}$ (D, D. A. Dongelmans and R. J. de Haan
}

(C) 2016 Springer-Verlag Berlin Heidelberg and ESICM

\section{Dear Editor,}

We thank Dr. Ligtenberg and colleges for their interest in our paper [1] and their subsequent correspondence [2], in which they comment on whether results of our study indicate that transports of critically ill patients in stable hemodynamic and respiratory condition with a mobile intensive care unit (MICU) can be safely performed by a team with a critical care nurse and a paramedic.

We agree that a MICU represents a setting with limited personnel resources in the case the occurence of a critical event. Thereby, the authors' concern on the safety issue is well taken and indeed reflects the rationale of this trial.

We would like to stress that the objective of our study was not to detect a difference between the outcomes of two transport options using a classical superiority trial design, but to establish whether the omission of a critical care physician during MICU transports does not worsen the current transport protocol, which includes a physician. To answer this research question the use of a noninferiority trial design is the appropriate choice.

However, a point in case was the higher rate of critical events we found, which contrasted with the lower rate we assumed at the time of the sample size calculation. Consequently, as we have noted in our Discussion section, our trial turned out to be too small to unequivocally establish non-inferiority of nurse-led transports.
We therefore concluded, in line with the authors' opinion, that until better evidence is available no changes in the present transport policy should be made. Nevertheless, we believe that if a validated triage method were to be available, it is possible that a nurse-based team would perform as well as a nurse/physician-based team. A prerequisite to such an outcome, however, is the selection of patients in a stable hemodynamic and respiratory condition. We advocate the development and testing of such criteria as a next step.

Compliance with ethical standards

Conflicts of interest

On behalf of all authors, the corresponding author states that there is no conflict of interest.

Accepted: 22 August 2016

Published online: 12 September 2016

References

1. van Lieshout EJ, Binnekade J, Reussien E, Dongelmans D, Juffermans NP, de Haan RJ, Schultz MJ, Vroom MB (2016) Nurses versus physician-led interhospital critical care transport: a randomized non-inferiority trial. Intensive Care Med 42:1146-1154

2. Ligtenberg J, Kruger HL, Zijlstra JG (2016) Interhospital critical care transports: have a safe trip! Intensive Care Med. doi:10.1007/ s00134-016-4499-9 